\title{
Protocolo de intervención integral para hombres con trastornos depresivos graves y crónicos en una unidad de salud mental comunitaria.
}

Comprehensive intervention protocol for men with major and chronic depressive disorder.

Rafael Romero Gamero ${ }^{\mathrm{a}}$, Nicolás Vucínovich ${ }^{\mathrm{b}}$, Silvia Poves Oñatec

${ }^{a}$ Psicólogo Clínico. Unidad de Salud Mental Comunitaria-Oriente. Hospitales Universitarios "Virgen del

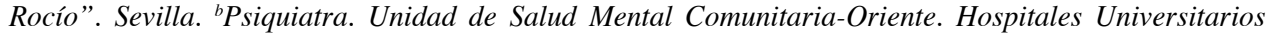
"Virgen del Rocío". Sevilla. 'Psicóloga Clínica. Centro de Salud Mental de Aranjuez. Orden Hospitalaria San Juan de Dios. Madrid.

Correspondencia: Rafael Romero Gamero (rrgamero@yahoo.es)

RESUMEN: Introducción: Los varones con cuadros depresivos presentan mayor incidencia de suicidio, de consumo de drogas y alcohol, mayores niveles de irritabilidad y una tendencia más acusada a negar la ayuda profesional. Lo anterior sitúa a la población masculina en un estado de peor pronóstico. Por lo que una pronta detección e intervención se convierte en un objetivo prioritario.

Método: Se ha confeccionado un protocolo en tres fases: 1) valoración integral inicial psicopatológica y funcional, 2) intervención combinada tanto psicológica (grupal e individual) como farmacológica en una perspectiva comunitaria, con una participación activa por parte del paciente y familia, para ofrecer el abordaje más multidisciplinar y efectivo posible cuya duración llega a los dos años; y 3) seguimiento durante un año para evitar recurrencias.

Resultados: los datos obtenidos y la impresión clínica indican una importante disminución de la sintomatología depresiva y de mejora en su nivel de funcionamiento general. Conclusión: el protocolo descrito nos permite tener organizado de antemano nuestro trabajo cotidiano aprovechando de forma productiva los recursos de que disponemos.

PALABRAS CLAVES: Trastorno depresivo, Hombres, Trastorno Distímico, Psicoterapia, Antidepresivos.

\section{Introducción}

A pesar de la respuesta favorable a los tratamientos psicológicos y farmacológicos que presentan los cuadros depresivos así como el amplio arsenal terapéutico con el que contamos en la actualidad, un porcentaje importante de pacientes tiene una evolución crónica (1) (2), llegando al 20\% los pacientes que no presentan una evolución de recuperación total, con una elevada sintomatología residual y que son

Recibido: 06/09/2009; aceptado: 30/09/2009

Rev. Asoc. Esp. Neuropsiq., 2010; 30 (106), 249-261.
ABSTRACT: Introduction: Males with depressive disorders have a higher incidence of suicide, drugs and alcohol use, a higher level of general anger and they often refuse to professional treatment as well. They are included in worse prognosis population. That is the reason why fast detection and treatment is the main priority.

Method: This study describes an intervention protocol developed through three phases: 1) comprehensive psychopathological and functional evaluation, 2) combination of different types of psychological treatment (group and individual) as well as pharmacological treatment. This intervention protocol is based on a communitarian vision of Mental Health. That includes an active participation of patients, their families and professionals due to an effective treatment two years long. 3) monitoring one year long to prevent relapses.

Results: data found and clinical global impression show a relevant decreased of depressive symptoms and improve on functional capabilities. Conclusion: our intervention protocol allows us organize psychotherapeutic treatment in order to reach a higher efficiency.

KEY WORDS: Depressive disorder, Male, Dysthymic disorder; Psychotherapy; Antidepressive Agents. 
refractarios a tratamientos. Se ha llegado a manejar cifras en la población general de hasta el 5\% de prevalencia (3). La comorbilidad con otros trastornos como los de tipo ansioso suele ser la tónica más que la excepción (4). La comorbilidad con trastornos de la personalidad tanto del grupo B como del C también es muy elevada (5). Se observa una mayor repercusión en el funcionamiento social, familiar y laboral respecto a la observada en los cuadros agudos (6).

Por consiguiente el elevado porcentaje de pacientes con trastornos depresivos con una duración superior a los 18 meses ha llevado a insistir en la consideración de la depresión como un trastorno con alto riesgo de cronicidad (7). A nivel conceptual ha supuesto que se hable cada vez más de las depresiones crónicas, un subgrupo compuesto de cuadros ya existentes en las clasificaciones actuales (7) (8). Nos referimos al trastorno distímico, la depresión mayor en remisión parcial, el trastorno depresivo recurrente y el trastorno depresivo mayor subtipo crónico.

Aunque en toda la inmensa mayoría de estudios epidemiológicos la prevalencia en la población femenina es superior a la detectada en la masculina (9), la depresión en los hombres presenta unas características que le hace merecer una especial atención. Uno de los aspectos más alarmantes es el alto nivel de suicidio entre hombres deprimidos en comparación a las mujeres llegando a ser hasta cuatro veces más frecuente (10), a pesar de que las mujeres lo intentan más. Los métodos usados por ellos son más letales. También se observa una menor tendencia a solicitar ayuda profesional lo que podría redundar en la mayor tasa de suicidios consumados. El perfil sintomático de los hombres se caracteriza por una mayor fatiga, irritabilidad, pérdida de aliciente por el trabajo o tiempo libre y de problemas de sueño, y no presentan, al contrario de las mujeres, tantos síntomas relativos a sentimientos de tristeza, falta de autoestima y culpabilidad excesiva (11), síntomas, estos últimos más relacionados con la imagen social sobre lo que es la depresión. Estas características pueden determinar las diferencias en su detección por género. Algunos autores valoran el mayor abuso de alcohol y drogas como un posible "síntoma" de depresión en hombres (12). En lugar de pedir ayuda directamente, permitiéndose verbalizar su malestar, los hombres pueden intentar reducirlo abusando de drogas y alcohol, en especial a la hora de afrontar determinados sentimientos como la irritabilidad, frustración o tristeza. Otros tienden a refugiarse en el trabajo o mostrando conductas impulsivas poniéndose en situaciones peligrosas (11) como la conducción temeraria.

No hay que olvidar la influencia de los factores culturales y sociales que atribuyen a los hombres un rol deseable de "fortaleza", viéndose la depresión como un estigma relacionado con una debilidad o incapacidad de autocontrol, lo cual le puede llevar a canalizar los sentimientos de tristeza a través de conductas de hostilidad, una reacción más "masculina". Por otro lado se observa que las mujeres responden mejor a los tratamientos farmacológicos con ISRS (13) y, en cuanto a la psicoterapia, los hombres presentan menor nivel de cumplimento del plan terapéutico. 
ORIGINALES Y REVISIONES

Por otro lado, la combinación de tratamientos ha repercutido en mejores resultados en comparación con el tratamiento solo farmacológico o psicológico (14). Respecto a la evidencia existente se observa que el tratamiento farmacológico produce un alivio relativamente rápido de los síntomas agudos y el tratamiento psicológico produce una mejora en la adherencia a la medicación, mejora de las habilidades para afrontar situaciones estresantes, mejoría en sus relaciones sociales y, finalmente, una reducción más pronunciada de los síntomas residuales (15).

Todo lo anterior, nos ha llevado a prestar una especial atención a los hombres deprimidos y, en especial, con evoluciones poco favorables y crónicas que llegan a consultar a nuestra Unidad de Salud Mental Comunitaria y desarrollar un plan de actuación preferente por el alto riesgo de abandono, mala respuesta y riesgo autolítico.

Esto nos motivó a considerar determinados cambios en el enfoque general de actuación ante estos trastornos de evolución crónica (6) que supone a) un abordaje más prolongado en el tiempo que suponga no solo asistirles en la fase aguda sino en un periodo de continuación para evitar recaídas con un seguimiento clínico posterior y b) el uso conjunto de fármacos y psicoterapia para completar la eficacia de cada uno de los tratamientos en solitario (16).

A continuación exponemos nuestra experiencia durante los dos últimos años a la hora de detectar e intervenir sobre los hombres aquejados de depresiones graves y crónicas. Denominamos integral a la intervención propuesta ya que nuestro objetivo es ofrecer un abordaje lo más completo posible, según los recursos disponibles, y teniendo en cuenta las necesidades de pacientes y allegados según la perspectiva comunitaria que caracteriza nuestra labor cotidiana. El trabajo supone la participación de profesionales de la psicología clínica, psiquiatría y enfermería de salud mental así como otros profesionales si se estima conveniente (trabajadores sociales, etc). Implica una intervención que supone tanto una fase de valoración inicial completa, la puesta en práctica de tratamientos con apoyo empírico sobre su eficacia en trastornos depresivos y una etapa de continuación y seguimiento para evitar recaídas. Supone un trabajo en colaboración con miembros de su entorno más inmediato como son sus familiares y parejas. Finalmente tenemos en cuenta el uso de otros dispositivos como la Unidad de Rehabilitación (U.R.A.) o Unidad de Hospitalización de Salud Mental dependiendo de las necesidades de los pacientes. Todo ello organizado en un plan de intervención sistematizado que aumente, como hemos comentado, la eficiencia de nuestro trabajo cotidiano.

Protocolo de actuación ante hombres con trastornos depresivos graves y crónicos.

El plan de actuación que proponemos va dirigido a hombres que cumplen los criterios siguientes:

- Un diagnóstico de depresión de larga evolución y de gravedad como, por 
ejemplo: depresión mayor en remisión parcial, trastorno depresivo recurrente y el trastorno depresivo mayor, subtipo crónico.

- Por otro lado, se dará preferencia a los afectados que no hayan respondido a los tratamientos psicoterapéuticos o/y farmacológicos que han recibido hasta el momento actual.

- Pacientes con ideación autolítica o importante alteración en su funcionamiento familiar, social y laboral.

En resumen, hombres con cuadros depresivos cuya gravedad, cronicidad y falta de respuesta a tratamientos habituales (psicológicos y farmacológicos) precisen de un abordaje más específico y completo. Un total de 25 pacientes se han beneficiado del protocolo.

El plan supone tres etapas:

A) Valoración integral inicial, que conlleva el establecimiento de la alianza terapéutica y la conclusión del plan de tratamiento individualizado según sus características y necesidades.

B) Puesta en práctica del plan terapéutico consensuado.

C) Etapa de seguimiento tras mejoría o derivación a otros dispositivos asistenciales en caso de no mejorar.

A. Valoración inicial. Cuando se detecta un posible usuario candidato a ser incluido en el plan de actuación se inicia una primera fase de valoración consistente en varias entrevistas:

1) Interconsulta con el otro facultativo del programa (psicólogo clínico/psiquiatra). Supone una primera toma de contacto con el otro miembro del equipo, que llevara la parte complementaria del tratamiento, para valorar las necesidades de tratamiento psicológico o farmacológico dependiendo del caso. El objetivo de estas primeras entrevistas es que sea valorado para recibir un tratamiento lo más completo, multidisciplinar y adecuado a las necesidades del afectado ateniéndonos a las recomendaciones actuales que plantean la combinación de medicación y psicoterapia como el mejor tratamiento para la depresión grave, crónica o resistente (14).

2) Entrevistas de valoración de su estado psicopatológico y funcional a través del uso de pruebas psicológicas (cuestionarios y escalas). Las pruebas usadas por protocolo son las siguientes:

a. Para valorar el nivel de depresión y posteriormente la respuesta a los tratamientos hemos decidido usar la escala MADRS (Montgomery-Asberg Depression Rating Scale) ampliamente usada en la valoración de la depresión a nivel de estudios epidemiológicos y clínicos (17).

b. El Inventario de Discapacidad de Sheehan (SDI) para recoger el nivel de discapacidad que el cuadro depresivo está provocando en distintas áreas de la 
ORIGINALES Y REVISIONES

vida de la persona (familiar, laboral y social), así como el nivel de estrés que está sufriendo y el nivel de apoyo que considera que está recibiendo (18).

c. Por último La Escala de Impresión Clínica (CGI-SI ), para analizar la impresión clínica de la gravedad del cuadro actual (19).

d. A parte de las medidas anteriores se pueden realizar otras según los objetivos particulares que nos planteemos ante cada paciente con depresión y atendiendo a su psicopatología actual: valoración de rasgos de personalidad a través del Cuestionario de Salamanca, valoración de su estado de ansiedad con la Escala de Ansiedad de Hamilton y, de su nivel de psicopatología global, con el Inventario de Síntomas (SCL-90R).

3) Se concreta con el paciente los objetivos que nos planteamos con la valoración que está recibiendo, motivándole al cambio tras una información detallada de las ventajas que supone, en consonancia con la evidencia científica, que reciba un tratamiento combinado. Se le explica el contenido del trabajo que se va a iniciar en caso de que decida participar y se consensúa con él el tipo concreto de tratamiento psicológico (grupal o individual) y farmacológico tras recibir toda la información pertinente.

4) Finalmente se tratan las expectativas, necesidades y temores de cada usuario ante la medicación y la intervención psicológica para solventar los obstáculos que determinadas ideas preconcebidas pueden suponer de cara al tratamiento.

Esta fase de valoración inicial tiene un carácter informativo y motivacional para, con una actitud comprensiva y empática, ir activando al paciente hacia una mayor responsabilidad de éste de cara al tratamiento en el cual se va a implicar y eliminar una imagen de receptor pasivo de actuaciones profesionales.

B. Plan terapéutico. La segunda fase es la de intervención propiamente dicha y comienza tras haberse concretado entre los facultativos y el paciente el plan de actuación personalizado. Reuniones periódicas de coordinación, entre el equipo de tratamiento mientras dura la intervención, servirán de intercambio de información e impresiones del proceso que lleva cada paciente permitiendo que se realicen las modificaciones pertinentes para mejorar nuestra labor asistencial. Ésta fase implica dos ramas de tratamiento paralelas, la psicológica y la farmacológica, que comentamos a continuación:

\section{Rama de tratamiento psicológico}

Tras la valoración psicométrica y de necesidades el paciente decide iniciar un tratamiento psicológico siendo la primera opción la intervención grupal. Entre sus ventajas están las de compartir experiencias semejantes y una búsqueda más constructiva de soluciones a su problemática actual que está originando o manteniendo su estado depresivo. Han estado presentes, desde el principio, el cuestionamiento por parte de los propios pacientes de los aspectos culturales y sociales relacionados 
con el género masculino que han influido en su estado actual: qué les ha supuesto ser el "cabeza de familia", el significado personal sobre la expresión abierta de emociones, el temor a la debilidad, el valor dado al trabajo como forma de realizarse, etc.

Nos hemos decantado por el uso de la Psicoterapia Interpersonal en grupo. Su demostrada eficacia en cuadros depresivos (20), las características tanto de estructura como de contenido de éste abordaje terapéutico, unido a su carácter ecléctico en el uso de técnicas, posibilita un mayor margen de maniobra a la hora de buscar las soluciones previamente comentadas. Las sesiones son de 90 minutos con una periodicidad semanal al principio y que irán pasando a quincenales y en la última etapa, mensuales. La duración total será de 2 años, tiempo más que suficiente para abarcar un abordaje en fase aguda y otra de continuación para reforzar las mejorías y prevenir recaídas.

En caso de que el paciente no haya aceptado la psicoterapia grupal pasa a una psicoterapia individual de sesiones mensuales, que irán acompañadas de contactos telefónicos periódicos para que el paciente pueda consultar cualquiera de las dudas sobre las pautas terapéuticas indicadas en las sesiones y que sirva de contención en momentos de crisis puntuales.

El abordaje individual está basado también en la Psicoterapia Interpersonal. La elección de esta terapia se basa en la semejanza de los motivos de consulta de la mayoría de los cuadros depresivos y los focos terapéuticos que se trabajan desde este enfoque (depresión por duelo, disputas interpersonales, falta de habilidades sociales y cambios vitales que suponen un cambio en sus roles sociales). Cuando existe una problemática relacional que influye claramente en el origen, mantenimiento o agravamiento del cuadro depresivo nos decantamos por este abordaje.

En los casos en que lo predominante es una clara inhibición psicomotriz, apatía, anhedonia y aislamiento social o cuando el usuario no desea profundizar en los aspectos relacionales de su depresión y tienen poco interés por un abordaje de introspección, hemos elegido un enfoque conductual, en especial basándonos en las aportaciones teóricas de Lewinsohn (21) (22), sobre la conducta depresiva como baja tasa de refuerzo positivo y un incremento de experiencias aversivas. Para ello nos hemos centrado en un programa de incremento de conductas gratificantes y el desarrollo de las habilidades necesarias para conseguirlas (habilidades sociales, cambios ambientales, manejo del tiempo, relajación, etc). Los resultados de los últimos estudios sobre los componentes eficaces de la Terapia Cognitiva, donde se ha observado que la eficacia del componente conductual es equivalente a todo el tratamiento completo, defiende el uso de éstas estrategias en depresiones severas (23). En algunos casos la combinación de elementos de ambos abordajes, tanto el interpersonal como el conductual, ha sido especialmente útil. 
ORIGINALES Y REVISIONES

Finalmente no debemos olvidar la importancia que está adquiriendo, tanto a nivel teórico como práctico, la Psicoterapia Analítico-Funcional (PAF) a la hora de conceptualizar el trabajo de la relación terapéutica y su uso para reforzar la eficacia de otras intervenciones (24). Sirve de puente entre conceptos psicoanalíticos y conductuales, reformulando muchos tópicos psicoanalíticos desde la perspectiva del conductismo skinneriano. Se centra en atender a las conductas (verbales y no-verbales) consideradas clínicamente relevantes que surgen durante las sesiones terapéuticas, tanto las indicativas de su problemática interpersonal como las que sugieren avances, con el objetivo de irlas reforzando diferencialmente para un cambio paulatino. Supone tener en cuenta unas series de reglas como: atender a la ocurrencia de conductas clínicamente relevantes, su evocación, reforzar sus mejorías, prestar atención a la propia conducta y su influencia en la del paciente, y proporcionar interpretaciones que conecten lo que ocurre en las sesiones con otros contextos de su vida. Sus conceptos y reglas han estado presentes en todo momento, siendo en algunas ocasiones la principal intervención con la que hemos contado (24).

\section{Rama de tratamiento psicofarmacológico:}

En cuanto al tratamiento farmacológico de los pacientes el recorrido realizado es el siguiente (25) (26):

Si existen antecedentes de buena respuesta a un fármaco antidepresivo determinado, incluso entre familiares de primer grado, se elige habitualmente el mismo antidepresivo.

1. Como primera opción comenzamos con un ISRS, a dosis habituales. Se administran benzodiazepinas en casos de importante ansiedad o insomnio, buscando la reducción en 2 semanas y suspenderlas tras 4 semanas de tratamiento. Si se produce una remisión sintomática o mejoría significativa tras 4 semanas, se mantiene el tratamiento antidepresivo, en función del diagnóstico, durante 6 meses (hasta 1 año) en primeros episodios que no presenten un trastorno de la personalidad de forma concomitante; para segundos episodios y cuadros depresivos crónicos, el tratamiento se extenderá durante más de 1 año.

2. En caso de mejoría parcial tras 4 semanas, la primera opción será aumentar (duplicar la dosis de ISRS), manteniéndola si mejora de forma significativa.

3. Si tras 2 semanas de tratamiento no se observa mejoría parcial, se cambiará el ISRS por otro de la misma familia, aumentando la dosis en caso de mejoría parcial. Es importante, en caso de resistencia, revisar el diagnóstico y el conjunto del tratamiento, comprobar su cumplimiento y correcta dosificación.

4. En caso de no responder al segundo ISRS, sa optará por un antidepresivo dual tipo Venlafaxina de liberación prolongada, a dosis por encima de la media ( $>$ $\mathrm{o}=150 \mathrm{mg}$ ). Si mejora se mantendrá este mismo fármaco según se explica en el punto 1 . 
5. En caso de respuesta parcial o ninguna respuesta se aumentará la dosis hasta niveles máximos $(225-300 \mathrm{mg})$.

6. Si no obtenemos respuesta, la siguiente opción serán los antidepresivos tri-tetracíclicos (clomipramina, imipramina, nortriptilina, etc.) dosis superiores a $150 \mathrm{mg} /$ día (según la tolerancia y descartando ideación autolítica o antecedentes de sobreingesta farmacológica).

7. En caso de respuesta parcial o no respuesta se podrá combinar con Litio, buscando su efecto potenciador (también con metilfenidato, T4 u olanzapina). En casos de depresiones recurrentes, cuando el litio no es tolerado o está contraindicado, según nuestra experiencia la lamotrigina ha mostrado buenos resultados.

Las combinaciones de antidepresivos solemos practicarlas, por un lado, en casos de insomnio (trazodona, mirtazapina) aprovechando el efecto sedativo de estos fármacos conseguido a dosis en general bajas; $y$, por otro lado, en casos de resistencia, asociando antidepresivos de perfil bioquímico distinto. Entre estos últimos las asociaciones más frecuentes son: antidepresivos tri (tetra) cíclicos y los ISRS; ISRS y el bupropión, o entre la venlafaxina y la mirtazapina.

Por otro lado, como complemento al trabajo de los dos facultativos la participación del personal de enfermería es de gran ayuda. Se encarga de realizar seguimientos telefónicos y en caso de necesidad, cita al paciente para realizar el seguimiento del plan terapéutico instaurado tanto psicológico como farmacológico.

La intervención sobre el paciente ha sido completada con el trabajo con la pareja y familia para canalizar el estado emocional en que desemboca la convivencia con hombres aquejados de depresiones crónicas. Se pretende resolver las dudas, psicoeducar sobre la depresión y que vayan asumiendo un papel activo como aliados y aliadas del equipo terapéutico que repercuta en mejorar las relaciones de pareja y familiares influyendo, de este modo, positivamente en la evolución del cuadro depresivo.

En los casos que han recibido el protocolo de intervención integral y que no han presentado ninguna mejoría o refieren un agravamiento se plantearía una derivación a un tercer nivel para abordaje por parte de ellos, siendo la Unidad de Rehabilitación de Área el lugar elegido por los resultados positivos que se han obtenido, con la derivación de pacientes con patología depresiva resistente y crónica, en otras ocasiones. Desde que estamos aplicando nuestro protocolo ninguno de los pacientes que han formado parte de él ha precisado su derivación. Así mismo, no hemos precisado programar un internamiento en la Unidad de Hospitalización de Salud Mental.

C. Seguimiento. Finalmente, tras las fases de valoración integral inicial y de puesta en práctica del plan de tratamiento consensuado, los pacientes entran en una tercera etapa de seguimiento con consultas programadas durante el año siguiente 
ORIGINALES Y REVISIONES

de concluir la fase de tratamiento activo. El objetivo que se persigue es evaluar su estado psicopatológico, el funcionamiento psicosocial y estar atentos a posibles complicaciones que surjan en su contexto interpersonal que puedan precipitar otro episodio depresivo, actuando en el menor tiempo posible a través del refuerzo de la puesta en práctica, por parte del paciente, de los aprendizajes obtenidos en su periodo de psicoterapia. (Gráfico 1)

\section{Resultados}

Durante los dos años que lleva funcionando nuestro protocolo de actuación los resultados han sido positivos: se ha evitado el ingreso hospitalario de varios pacientes, han presentado mejoría clínica general con reducción paulatina del tratamiento farmacológico, un incremento de su funcionamiento social y de calidad de vida.

Actualmente contamos con datos de resultados pre-postratamiento del grupo de psicoterapia, la valoración a través de las escalas, que hemos aplicado como protocolo a todos los pacientes (MADRS, SDI y CGI-SI), han demostrado una mejoría significativa y mantenida de la sintomatología depresiva, más concretamente todos han presentado una reducción de su sintomatología superior al $45 \%$, cuatro de ellos con sintomatología reducida en más del 50\%, por tanto, podemos afirmar, que ha habido una buena respuesta al tratamiento y en dos de ellos podemos decir que se ha producido una remisión clínica.

Se ha producido una mejoría muy significativa en el nivel de discapacidad tanto a nivel familiar y social, considerando los pacientes que encontraban extremadamente afectadas ambas áreas al principio de la asistencia al grupo. Sus puntuaciones posteriores han descendido a niveles de afectación moderada o, incluso leve, en algún caso.

Respecto al nivel de estrés que consideran sufrir, lo valoraban como extremo al principio, siendo al final valorado en unos niveles moderados y más manejables. Se observa un aumento de su nivel de apoyo social que no presentaban en muchos años.

La impresión clínica a través del CGI-SI confirma estas mejorías. Han pasado como grupo, a una puntuación indicativa de encontrarse "grave o extremamente enfermos" a un nivel de "levemente enfermos".

Finalmente, los miembros refieren encontrarse más cómodos en su expresión de sentimientos y han disminuido los temores y prejuicios que tenían ante ello. Se sienten más dispuestos a solicitar y recibir ayuda de forma abierta sin que ello suponga un menoscabo en su autoestima. 


\section{Discusión}

Las propias características de estos pacientes (la elevada sintomatología depresiva, la preocupación por la valoración de los demás, la propia inhibición psicomotora de la depresión, la constante tendencia a la focalización en su malestar, etc.,), podría haber impedido una adecuada adherencia al tratamiento combinado. Sin embargo, consideramos que la valoración inicial a través de las entrevistas individuales, con un adecuado análisis de las necesidades y expectativas de los pacientes, ha posibilitado una mejor adherencia de los pacientes al trabajo terapéutico eliminando las ideas preconcebidas y temores que pudieran influir en un abandono precipitado.

Aunque el trabajo en equipo debe de ser la tónica habitual en las distintas Unidades de Salud Mental Comunitaria, así como en el resto de dispositivos de Salud Mental, en ocasiones no es así. La elevada presión asistencial, en especial, de trastornos adaptativos o patologías denominadas menores que unido a una visión simplificadora de la patología, del sufrimiento del paciente y del entorno (biologización o psicologización excesiva) y la existencia de unas inadecuadas relaciones profesionales o personales entre distintos miembros de los dispositivos, puede desembocar en una carencia de trabajo en equipo con la consecuente repercusión negativa en el eslabón más débil de la cadena que son los usuarios y usuarias, en especial, los afectados de patologías más graves e incapacitantes.

Por todo ello sería adecuado no perder de vista nuestra labor en equipo a través de la instauración de planes de actuación ante determinadas patologías que exigen una intervención eficaz y eficiente a través de un trabajo integral y de colaboración entre distintos profesionales.

El protocolo de intervención que hemos presentado ante hombres con depresiones graves y crónicas nos permite tener organizado de antemano nuestro trabajo cotidiano aprovechando de forma productiva los recursos de que disponemos y sacando el máximo de ellos. Hasta el momento los resultados obtenidos refuerzan seguir en ésta línea. 
GRAFICO 1: FASES DEL PROTOCOLO

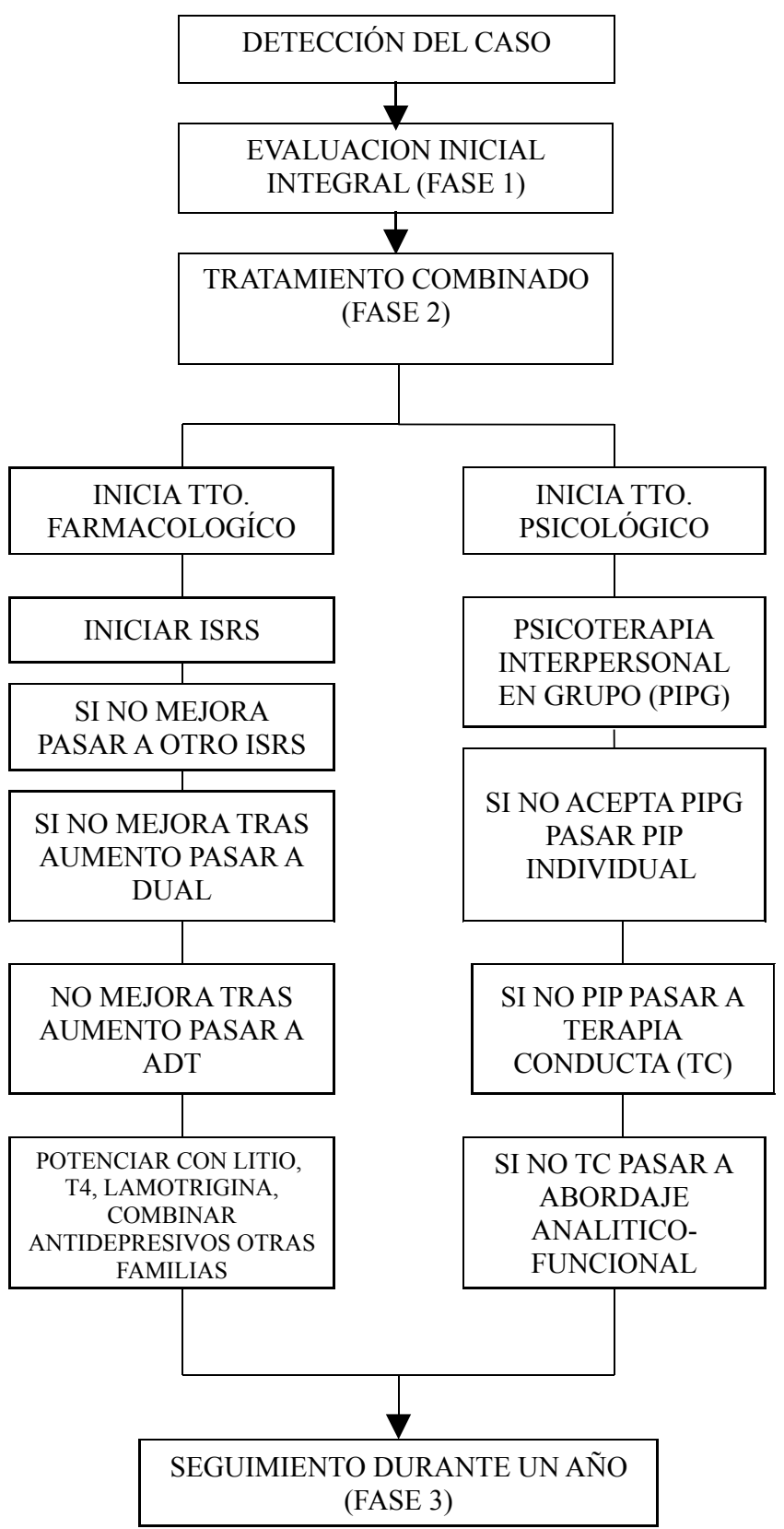




\section{BIBLIOGRAFÍA:}

(1) Elkin, I.; SheA, M.T., Watkins, J.T. Imber, S.S., Sotsky,S.M., Collins, J.F., ET AL. “Treatment of Depression Collaborative Reseach Program. General Effectiveness of treatments. N.I.M.H", Archives of General Psychiatry, 1989, 46, pp. 973-982.

(2) Shea, M.T., Elkin, I., Imber, S.D. Sotsky, S.M., Watkins, J.T., Collins, J.F., ET Al.” Course of depression symptoms over follow up. Findings from the National Institute of Mental Health Treatment of Depression collaborative Reseach Program" Archives of General Psychiatry, 1992, 49, pp. 782-787.

(3) Gastó, C., Vallejo, J. Y Menchón, J.M., Depresiones crónicas, Barcelona, Doyma, 1993.

(4) Browne, G., Steiner, M., Roberts, J., Gafni A., B yrne, C. Dunn E., et Al. (1999), "Prevalence of dysthymic disorders in primary care”, Journal of Affective Disorders, 1999, 54, pp. 303-308.

(5) Pepper, C.M., Klein, D.N., Anderson, R.L. Riso, L.P., Ouinette, P.C. LizARdi, H. "DSMIII-R Axis II comorbility in dysthymia and major depression", American Journal Psychiatry, 1995, 152 pp. 239-247.

(6) Klein, N.D. y Santiago, N.J., "Dysthymia and Chronic Depression: Introduction, Classification, risk Factors and Course", Journal of Clinical Psychology, 2003, 59, pp. 807-816.

(7) American Psychiatric Association, Diagnostic and Statistical Manual of Mental Disorders, 4th edition,Text Revision. Washington DC, American Psychiatric Association, 2000.

(8) Organización Mundial de la Salud, La Clasificación CIE-10 de los Trastornos Mentales y del Comportamiento: Criterios diagnósticos de investigación, OMS, Ginebra, 1993.

(9) Weissman, M.M., Bland, R.C.,Canino, C.J., Faravelli, C., Greenwald, S.; Hai-Gwo Hwu, ET AL. "Cross-national epidemiology of major depression and bipolar disorder", Journal of the American Medical Association, 1996, 276, pp. 293-299.

(10) Minino Am, Arias, E., KochaneK, K.D., Murphy, S.L., Smith, B.L., Deaths: final for data for 2000. National Vital Statistics Reports; 50(15), Hyattsville, MD, National Center for Health Statistics, 2002.

(11) Cochran, S.V., Rabinowitz, F.E. Men and depression: clinical and empirical perspectives, San Diego, Academic Press, 2002.

(12) Robins, L.,Regier, D. Psychiatric disorders in America, New York, Free Press, 1991.

(13) Konnstein, S.G. "Gender differences in depression: implications for treatment", Journal of Clinical Psychiatry, 1997, 58, pp.12-18.

(14) Keller, M.B., McCullough, J.P. Klein, D.N., Arnow, B., Dunner D.L., Gelendberg A.J., ET AL., "A comparison of Nefazodone, the Cognitive Behavioral-Analysis System of Psychotherapy, and their combination for treatment of chronic depression" New England Journal of Medicine, 2002, 342, pp. $1462-1470$.

(15) Thase, M.E., Greenhouse, J.B., Frank, E., Reynolds,C.F,III, Pilkonis, P.A., Hurley, K., ET AL. "Treatment of major depression with psychotherapy or psychotherapy-pharmacotherapy combinations", Archive of General Psychiatry, 1997, 54, pp.1009-1015.

(16) Arnow, B.A. y Constantino, M.J. "Effectiveness of Psychotherapy and combination Treatment for Chronic Deression”. Journal of Clinical Psychology, 2003, 59, pp. 893-905.

(17) Montgomery, S.A y Asberg, M. "A new depression scale dessigned to be sensitive to change”, British Journal of Psychiatry, 1979, 134, pp.382-289.

(18) Sheehan, D.V., Hamett-Sheehan, K., y RaJ, B.A. "The measurement of diseability", International Clinical Psychopharmacology, 1996, 11(3), pp.89-95. 
ORIGINALES Y REVISIONES

(19) Guy, W., Early Clinical Drug Evaluation (ECDEU) Assessment Manual. Rockville, National Institute Mental Health, 1976.

(20) Klerman, G.L., Weissman, M.M, Rounsanville, B.J., y Chevron, E.S., Interpersonal Psychotherapy of Depression, New York, Basic Books,1984, pp. 71-182.

(21) Lewinsohn, P.M., Sullivan, J.M., Y Grosscup, S.J. "Changing reinforcing events: An approach to the treatment of depression", Psychotherapy: Theory, Reseach, and Practice, 1980, 47, pp.322334.

(22) JoRquera, A., GuARCH, J., (COORD.).Tratando...trastorno distímico y otros trastornos depresivos crónicos, Madrid, Pirámide, 2006.

(23) Dimidjan, S. Hollon, S.D., Dobson, K.S.; Schmeling, K.B.; Kohlenberg, R.J.; AdDis, et AL., "Randomized trial of Behavioral Activation, Cognitive Therapy and antidepressant medication in the acute treatment of adult", Journal of Consulting and Clinical Psychology, 2006, 74(4), pp.658-670.

(24) Kotlenberg, R. Y Tsai, M., Funtional Analitic Psychotherapy: creating intense and curative therapeutic relationships, New York, Plenum,1991

(25) Palomo, Jiménez-Arriero, Manual de Psiquiatría, Madrid, 2009.

(26) Soler Insa, Gascon Barrachina. Recomendaciones Terapéuticas en los Trastornos Mentales, Barcelona, Ars Medica, 2005. 